Mini Review

\title{
On the Van der Waals equation
}

\section{Abstract}

It is shown that the usual derivations of the van der Waals equation are inconsistent. The van der Waals equation is derived here in a more general framework, which employs the mean-field approximation.

PACS: 05.20.Gg; 51.30.+1; 0570.Ce

Keywords: Van der Waals equation, non-ideal gases, equation of state
Volume I Issue 5 - 2018

\section{MApostol}

Department of Theoretical Physics, Institute of Atomic Physics, Romania

Correspondence: M Apostol, Department of Theoretical Physics, Institute of Atomic Physics, Magurele-Bucharest MG-6, PO Box MG-35, Romania, Email apema@theory.nipne.ro

\section{Introduction}

Let us consider a classical gas of $\mathrm{N}$ identical point like particles enclosed in a volume $\mathrm{V}$ with the interaction energy

$$
E=\frac{1}{2} \sum_{i \neq j} \mathrm{U}\left(\mathbf{r}_{i}-\mathbf{r}_{j}\right)
$$

where $\mathrm{U}\left(\mathbf{r}_{i}-\mathbf{r}_{j}\right)$ is the two-particle interaction and $\mathrm{i}, \mathrm{j}=1,2, \ldots \mathrm{N}$ denote the particles. For point like particles $\mathrm{U}\left(\mathbf{r}_{i}-\mathbf{r}_{j}\right)$ is a function only of $\left|\mathbf{r}_{i}-\mathbf{r}_{j}\right|$.The correction to the free energy is given by

$$
\begin{gathered}
\Delta F=-T \ln \left(\frac{1}{V^{N}} \int e^{-\beta E} d \mathbf{r}_{1} . . d \mathbf{r}_{N}\right)= \\
=-T \ln \left[\frac{1}{V^{N}} \int\left(e^{-\beta E}-1\right) d \mathbf{r}_{1} . . d \mathbf{r}_{N}+1\right],
\end{gathered}
$$

where $T=1 / \beta$ is the temperature. We assume that the interaction $\mathrm{U}\left(\mathbf{r}_{i}-\mathbf{r}_{j}\right)$ is short range (integrable) and, moreover, it proceeds by collisions. More, we assume that the amount of gas is so small, that only one collision takes place at one time; since the free energy is a function of the form $F=N f(T, V / N)$, it follows that the result will be valid for any amount of gas. In addition, we neglect the multiparticle collisions, as for a rarefied gas. In these conditions equation (2) can be written as

$$
\begin{aligned}
\Delta F & =-T \ln \left[\frac{N(N-1)}{2 V^{2}} \int\left(e^{-\beta U\left(\mathbf{r}_{1}-\mathbf{r}_{2}\right)}-1\right) d \mathbf{r}_{1} d \mathbf{r}_{2}+1\right] \simeq \\
& \simeq-T \ln \left[1-\frac{N^{2}}{2 V^{2}} \int\left(1-e^{-\beta U\left(\mathbf{r}_{1}-\mathbf{r}_{2}\right)}\right) d \mathbf{r}_{1} d \mathbf{r}_{2}\right]
\end{aligned}
$$

if the amount of gas is still sufficiently large (such as to neglect the surface effects), we may write

$$
\begin{gathered}
\Delta F=-T \ln \left(1-\frac{N^{2}}{V} B\right), \\
B=\frac{1}{2} \int\left(1-\mathrm{e}^{-\beta U(\mathrm{r})}\right) d \mathbf{r} .
\end{gathered}
$$

The interaction $\mathrm{U}(\mathrm{r})$ is very repulsive for small $\mathrm{r}$ and very small in comparison with $\mathrm{T}$ at large distances; consequently, we may approximate the parameter $\mathrm{B}$ as

$$
\begin{gathered}
B=b+a / T,(6) \\
b=2 \pi \int_{0}^{2 r} d r \cdot r^{2}, a=2 \pi \int_{2 r_{0}} d r \cdot r^{2} U(r),
\end{gathered}
$$

$2 r_{0}$ being a scale distance which separates the "hard-core" part of $\mathrm{U}(\mathrm{r})$. From equation (4) we get the correction to pressure

$$
\Delta p=\frac{T \frac{N^{2}}{V^{2}} B}{1-\frac{N^{2}}{V} B}(8)
$$

and the total pressure

$$
p=\frac{N T}{V}+\frac{T \frac{N^{2}}{V^{2}} B}{1-\frac{N^{2}}{V} B}=\frac{N T}{V}\left(1+\frac{\frac{N}{V} B}{1-\frac{N^{2}}{V} B}\right)
$$

or

$$
p=\frac{N T}{V} \frac{1-\frac{N^{2}}{V} B(1-1 / N)}{1-\frac{N^{2}}{V} B}
$$

Since $\mathrm{N}$ is still much larger than 1 , we get $p=N T / V$, which shows that the interaction does not change the equation of state of the ideal gases.

It is customary to use the approximation

$$
\begin{aligned}
p & =\frac{N T}{V}\left(1+\frac{\frac{N}{V} B}{1-\frac{N^{2}}{V} B}\right) \simeq \frac{N T}{V}\left(1+\frac{N B}{V}\right)= \\
& =\frac{N T}{V}\left(1+\frac{N B}{V}\right)+\frac{N^{2} a}{V^{2}} \simeq \frac{N T}{V-N b}+\frac{N^{2} a}{V^{2}},
\end{aligned}
$$

which leads to the van der Waals equation. ${ }^{1}$

$$
\left(p-\frac{N^{2} a}{V^{2}}\right)(V-N b)=N T
$$

(written, usually, with $a<0$, as for attractive interaction at large distances. However, if we neglect $N^{2} B / V \ll 1$ in the denominator of equation (11), we should also neglect $N B / \mathrm{V}=\left(N^{2} B / V\right) / N \ll N^{2} B / V \ll 1$ in the numerator, which removes any effect of the interaction in the final formula. 
The difficulties related to the van der Waals equation have been discussed previously. ${ }^{2}$ The expansion of the thermodynamic potential $\Omega=-p V$ in powers of $\xi=\left(m T / 2 \pi \hbar^{2}\right)^{3 / 2} e^{\mu / T} \ll 1$, where $\mu$ is the chemical potential (and $\hbar$ is Planck's constant), ${ }^{3}$ exhibits the same drawback; indeed, we $\Omega=-T \ln (1+\xi V+\ldots$.$) and N=V \xi+\ldots$, such that $\Omega=-p V=-T \ln (1+\mathrm{N}$....) which is written usually as $\Omega=-p V=-T \ln (1+\mathrm{N} \ldots ..) \simeq-N T$; however, this approximation is valid for $N \ll 1$, a condition which is not satisfied.

The difficulties with the usual derivations of the van der Waals equation reside in the fact that an ensemble of interacting particles are not statistically independent; consequently, we need to solve first the problem of interaction. First, we leave aside correlations (which may lead to sound waves in a gas (collective excitations), which contribute to the statistical properties, though to a lesser extent); then, from equation (1) we see that each particle $i$ is acted by the rest of the particles by a potential

$$
\Phi\left(\mathbf{r}_{i}\right)=\sum_{j}^{\prime} U\left(\mathbf{r}_{i}-\mathbf{r}_{j}\right)
$$

where the prime means $\mathrm{j} \neq \mathrm{i}$; the total energy can be written as

$$
\mathrm{E}=\frac{1}{2} \sum_{i} \Phi\left(\mathbf{r}_{i}\right)
$$

The integration over $\mathbf{r}_{i}$ in equation (2) must take into account the requirement $j \neq i$, i.e., $\mathbf{r}_{j} \neq \mathbf{r}_{i}$ for any pairs $(i, j)$. It follows that the correction to the free energy is given by

$$
\Delta F=-T \ln \left(\frac{1}{V^{N}} \int e^{-\beta E} d \mathbf{r}_{1} \ldots d \mathbf{r}_{N}\right),
$$

where the prime means $\mathbf{r}_{j} \neq \mathbf{r}_{i}$. We perform this integration over a volume $V-N b$, where b is an "excluded" volume for each particle. In a mean-field approximation the potential $\Phi\left(\mathbf{r}_{\mathrm{i}}\right)$ does not depend on $\mathbf{r}_{i}$ and the summation in equation (13) is computed by

$$
\Phi=\sum_{j}^{\prime} U\left(r_{j}\right)=\frac{N}{V} \int_{2 r_{0}} d \mathbf{r} U(\mathrm{r})=\frac{2 N a}{V},
$$

where $b \simeq \frac{1}{2} .4 \pi\left(2 r_{0}\right)^{3} / 3$; the factor $1 / 2$ in estimating the volume $b$ arises from the fact that the independent integration with respect to any pair $\mathbf{r}_{\mathrm{i}}, \mathbf{r}_{j}$ counts twice the excluded volume. The change in the free energy becomes (equation (15))

$$
\begin{aligned}
& \Delta F=-T \ln \left(\frac{(V-N b)^{N}}{V^{N}} e^{-\frac{1}{2} \beta N \Phi}\right)= \\
& =\frac{1}{2} N \Phi-N T \ln \left(1-\frac{N b}{V}\right)=\frac{N^{2} a}{V}-N T \ln \left(1-\frac{N b}{V}\right) .
\end{aligned}
$$

We get the pressure

$$
\begin{aligned}
& p=\frac{N T}{V}+\frac{N^{2} a}{V^{2}}+N T \frac{N b / V^{2}}{1-N b / V}= \\
& =\frac{N T}{V}\left(1+\frac{N b / V}{1-N b / V}\right)+\frac{N^{2} a}{V^{2}}=\frac{N T}{V-N b}+\frac{N^{2} a}{V^{2}} ;
\end{aligned}
$$

hence, it follows immediately the van der Waals equation (12).
It is worth noting that we use the same cutoff $2 r_{0}$ to account for the prime in the integration in equation (15) and the prime in the potential given by equation (16). In general, we should employ two distinct cutoffs, but if the potential has a very sharp repulsive hard core the two cutoffs coincide. Also, we note that the mean-field approximation is equivalent with restricting ourselves only to oneparticle elementary excitations (quasi-particles). We have assumed that $\mathrm{a}$ and $\mathrm{b}$ do not depend on $\mathrm{V}$, and $\mathrm{b}>0$ (by its definition).

It may happen that an external potential $\varphi(\mathbf{r})$ is present and we wish to estimate its effects in the presence of the internal interaction. Then, the total energy is written as

$$
\mathrm{E}=\sum_{i} \varphi\left(\mathbf{r}_{\mathrm{i}}\right)+\frac{1}{2} N \Phi
$$

where $\Phi$ is a mean-field potential (also, the external potential may derive from a mean-field approximation). The ensemble has not translational symmetry anymore, since $\varphi(\mathbf{r})$ depends on position. The change in the free energy is

$$
\begin{aligned}
\Delta F & =-T \ln \left(\frac{1}{V^{N}} \int^{\prime} e^{-\beta \sum_{i} \varphi\left(\mathbf{r}_{\mathrm{i}}\right)-\frac{1}{2} N \Phi} d \mathbf{r}_{1} . . d \mathbf{r}_{N}\right)= \\
& =-T \ln \left[\left(\frac{1}{V} \int^{\prime} e^{-\beta \varphi(\mathbf{r})} d \mathbf{r}\right)^{N} e^{-\frac{1}{2} \beta N \Phi}\right]= \\
& =-N T \ln \left(\frac{1}{V} \int^{\prime} e^{-\beta \varphi(\mathbf{r})} d \mathbf{r}\right)+\frac{1}{2} N \Phi ;
\end{aligned}
$$

The integral in equation (20) can be written as

$$
\begin{gathered}
\int^{\prime} e^{-\beta \varphi(\mathbf{r})} d \mathbf{r}=\int e^{-\beta \varphi(\mathbf{r})} d \mathbf{r}-b \sum_{i} e^{-\beta \varphi\left(\mathbf{r}_{\mathbf{1}}\right)}= \\
=\int e^{-\beta \varphi(\mathbf{r})} d \mathbf{r}-\frac{N b}{V} \int e^{-\beta \varphi(\mathbf{r})} d \mathbf{r}=\left(1-\frac{N b}{V}\right) \int e^{-\beta \varphi(\mathbf{r})} d \mathbf{r},
\end{gathered}
$$

Such that we get

$$
\Delta F=-N T \ln \left(1-\frac{N b}{V}\right)-N T \ln \left(\frac{1}{V} \int e^{-\beta \varphi(\mathbf{r})} d \mathbf{r}\right)+\frac{1}{2} N \Phi
$$

we can see that we recover the van der Waals equation and get also the effects of the external field; for a finite ensemble, such effects may include surface contributions.

The general way of deriving the van der Waals equation consists in estimating the effects of the interaction in the mean-field equation (16) and taking account of the excluded volume, according to the definition of the interaction energy.

\section{Acknowledgements}

The author is indebted to the members of the Laboratory of Theoretical Physics at Magurele-Bucharest for many fruitful discussions. This work has been supported by the Scientific Research Agency of the Romanian Government through Grants 04-ELI / 2016 (Program 5/5.1/ELI-RO), PN 16420101 / 2016, PN 1909 01/2018 and PN (ELI) 164201 05/ 2016.

\section{Conflicts of interest}

Author declares that there is none of the conflicts. 


\section{References}

1. Van der Waals JD. Over de Continuiteit van den Gas- en Vloeistoftoestand. 1873. p. 1-154.
2. Cune LC, Apostol M. On the van der Waals equation. Roum J Phys. 2006;51(9-10):907-910.

3. Landau L, Lifshitz E. Course of Theoretical Physics. 3rd ed. Elsevier. $1980 ; 5$. 